\title{
FUNCTIONAL CALCULI AND DECOMPOSABILITY OF UNBOUNDED MULTIPLIER OPERATORS IN $L^{p}\left(\mathbb{R}^{N}\right)$
}

\author{
by ERNST ALBRECHT and WERNER J. RICKER*
}

(Received 16th September 1993)

\begin{abstract}
It is known, for each $1<p<\infty, p \neq 2$, that there exist differential operators in $L^{P}\left(\mathbb{R}^{N}\right)$ which are not (unbounded) decomposable operators in the sense of C. Foias. In this note we exhibit large classes of differential (and unbounded multiplier operators which are decomposable in $L^{p}\left(\mathbb{R}^{N}\right)$ and hence have good spectral mapping properties; the arguments are based on the existence of a sufficiently rich functional calculus. The basic idea is to take advantage of existing classical results on $p$-multipliers and use them to generate appropriate functional calculi.
\end{abstract}

1991 Mathematics subject classification: 47B40, 42B15.

\section{Introduction}

When investigating the global nature of linear differential operators with constant coefficients in spaces like $L^{p}\left(\mathbb{R}^{N}\right)$ an effective tool, when available, is the existence of a sufficiently rich functional calculus for the operator. Interpreting such operators as unbounded $p$-multiplier operators has the advantage of allowing the use of harmonic analysis techniques and shows the difficulties involved when $p \neq 2$. Since the range of the polynomial $Q$ determining the differential operator is always a subset of the spectrum of the operator, one cannot expect a large class of such operators to be infinitesimal generators of semigroups or integrated semigroups. Similarly, for $p \neq 2$, differential operators with constant coefficients are never spectral (in the sense of $N$. Dunford [4]) in the space $L^{P}\left(\mathbb{R}^{N}\right)$ except in the trivial case that $Q$ is constant [2]. Despite this negative aspect, there is a large class of operators which are not required to decompose the underlying $L^{p}$-space in such a strong way and whose members still enjoy the spectral mapping property; this is the class of those unbounded multiplier operators which are decomposable in the sense of $\mathrm{C}$. Foiaş.

It is shown in [2] that all (constant coefficient) elliptic differential operators are decomposable in $L^{p}\left(\mathbb{R}^{N}\right)$, for every $1<p<\infty$; the arguments are based on the existence of a sufficiently rich functional calculus. An example is given there of a (non-elliptic) differential operator which is not decomposable for every $p \neq 2$. The characterization of all (constant coefficient) differential operators which are decomposable seems to be a difficult task. The aim of this note is to present further classes of differential (and more general unbounded multiplier) operators which admit reasonable functional calculi and

*The support of an Alexander von Humboldt Fellowship is gratefully acknowledged. 
are decomposable. For instance, the generator of the Poisson semigroup, which corresponds to the unbounded multiplier $\xi \mapsto|\xi|$ in $L^{p}\left(\mathbb{R}^{N}\right)$, turns out to be decomposable, as does the wave operator

$$
\frac{\partial^{2}}{\partial x^{2}}-\frac{\partial^{2}}{\partial y^{2}}
$$

in $L^{p}\left(\mathbb{R}^{2}\right)$. The basic idea is to take advantage of existing classical results on p-multipliers (e.g. Mihlin, Marcinkiewicz, Littman-McCarthy-Rivière) and use them to generate appropriate functional calculi.

\section{Notation and preliminary results}

Given a Banach space $X$ we denote the space of all bounded linear operators in $X$ by $\mathscr{L}(X)$ and write $\mathbf{1}_{X}$ (or simply 1 ) for the identity operator on $X$. We recall that a Banach space has the Banach-Saks property (resp. weak Banach-Saks property) if every bounded sequence (resp. every weakly convergent sequence) has a subsequence whose arithmetic means are norm-convergent. Uniformly convex spaces (hence $L^{p}$-spaces for $1<p<\infty)$ have the Banach-Saks property [8] and $L^{1}$-spaces have the weak BanachSaks property [13]. The following fact is probably known; we include a proof for the sake of completeness.

Lemma 1.1. Let $X$ be a separable Banach space with the weak Banach-Saks property and let $\left(T_{n}\right)_{n=1}^{\infty}$ be a sequence in $\mathscr{L}(X)$ which converges to some operator $R \in \mathscr{L}(X)$ with respect to the weak operator topology. Then there is a sequence of operators $\left(S_{n}\right)_{n=1}^{\infty}$ converging to $R$ in the strong operator topology, where each operator $S_{n}$ belongs to the convex hull of the set $\left\{T_{n} ; n \in \mathbb{N}\right\}$.

Proof. Note that $K:=\sup \left\{\left\|T_{n}\right\| ; n \in \mathbb{N}\right\}$ is finite. Let $\left\{f_{n} ; n \in \mathbb{N}\right\}$ be a dense subset of $X$. By the weak Banach-Saks property, there exists a sequence $\left(T_{n}^{(1)}\right)_{n=1}^{\infty}$, whose members are the arithmetic means (and hence, finite convex combinations) of a subsequence of $\left(T_{n}\right)_{n=1}^{\infty}$, such that $T_{n}^{(1)} f_{1} \rightarrow R f_{1}$ in norm, as $n \rightarrow \infty$, and still $T_{n}^{(1)} \rightarrow R$, as $n \rightarrow \infty$, with respect to the weak operator topology. Moreover, $\sup \left\{\left\|T_{n}^{(1)}\right\| ; n \in \mathbb{N}\right\} \leqq K$. Using the weak Banach-Saks property we can find, by induction, sequences $\left(T_{n}^{(j)}\right)_{n=1}^{\infty}$, for each $j \in \mathbb{N}$, with the following properties.

(i) $T_{n}^{(j)} \rightarrow R$ in the weak operator topology, as $n \rightarrow \infty$.

(ii) Each operator $T_{n}^{(j)}$, for $j, n \in \mathbb{N}$, is a finite convex combination of operators in $\left\{T_{m} ; m \in \mathbb{N}\right\}$.

(iii) $\sup \left\{\left\|T_{n}^{(j)}\right\| ; n \in \mathbb{N}\right\} \leqq K$, for all $j \in \mathbb{N}$.

(iv) $T_{n}^{(j)} f_{k} \rightarrow R f_{k}$ in norm, as $n \rightarrow \infty$, for all $1 \leqq k \leqq j$ and $j \in \mathbb{N}$.

Now choose $S_{1} \in\left\{T_{n}^{(1)} ; n \in \mathbb{N}\right\}$ such that $\left\|S_{1} f_{1}-R f_{1}\right\|<2^{-1}$ and, inductively, 
$S_{j} \in\left\{T_{n}^{(j)} ; n \in \mathbb{N}\right\}$ such that $\left\|S_{j} f_{k}-R f_{k}\right\|<2^{-j}$ for all $1 \leqq k \leqq j$. It follows that $S_{j} f_{k} \rightarrow R f_{k}$, as $j \rightarrow \infty$, for all $k \in \mathbb{N}$, and that $\sup \left\{\left\|S_{j}\right\| ; j \in \mathbb{N}\right\} \leqq K$. Accordingly, $S_{j} \rightarrow R$ in the strong operator topology.

The set of all closed linear operators with domain $D(T)$ and range $\operatorname{ran}(T)$ contained in a given Banach space $X$ is denoted by $\mathscr{C}(X)$. If $T \in \mathscr{C}(X)$ and $Y$ is a closed subspace of $X$, then $Y$ is invariant for $T$ if $T(Y \cap D(T)) \subseteq Y$. The operator $T \mid Y$ with $D(T \mid Y):=Y \cap D(T)$ and defined by $(T \mid Y) y:=T y$, for every $y \in D(T \mid Y)$, is an element of $\mathscr{C}(Y)$. Recall that $T$ is decomposable in the sense of C. Foias (cf. [14]) if, for every finite open cover $\left\{U_{1}, \ldots, U_{r}\right\}$ of the one-point compactification $\overline{\mathbb{C}}:=\mathbb{C} \cup\{\infty\}$ of the complex plane, there are closed invariant subspaces $X_{1}, \ldots, X_{r}$ for $T$ such that $\sigma\left(T \mid X_{j}\right) \subseteq U_{j}$, for all $1 \leqq j \leqq r$, and $X_{1}+\cdots+X_{r}=X$. If $S \in \mathscr{C}(X)$ and $F$ is a closed subset of $\mathbb{C}$, we write $\mathscr{X}_{S}(F)$ for the set of all those $x \in X$ for which there exists an analytic $X$-valued function $f: \widetilde{C} \backslash F \rightarrow X$ such that $f(z) \in D(S)$ and $(z-S) f(z) \equiv x$ on $\mathbb{C} \backslash F$. Then $S$ has the LjubicMacaev property [11] if, for every locally finite open cover $\left(U_{j}\right)_{j=1}^{\infty}$ of $\mathbb{C}$ by bounded open sets, the space $X$ coincides with the closed linear span of the linear submanifolds $\mathscr{X}_{S}\left(\bar{U}_{j}\right), j \in \mathbb{N}$.

By $\mathscr{M}^{p}\left(\mathbb{R}^{N}\right)$ we denote the semisimple, unital, commutative Banach algebra of all $p$-multipliers on $\mathbb{R}^{N}$, for $1 \leqq p<\infty$, considered as essentially bounded functions on $\mathbb{R}^{N}$. Let $\mathscr{U}^{p}\left(\mathbb{R}^{N}\right)$ denote the set of all local $p$-multipliers on $\mathbb{R}^{N}$, that is, those measurable functions $\phi: \mathbb{R}^{N} \rightarrow \mathbb{C}$ with the property that $\rho \phi \in \mathscr{M}^{p}\left(\mathbb{R}^{N}\right)$ whenever $\rho \in \mathscr{C}_{c}^{\infty}\left(\mathbb{R}^{N}\right)$.

Let $1<p<\infty$ and fix $\psi \in \mathscr{U}^{p}\left(\mathbb{R}^{N}\right)$. Let $\mathscr{D}^{p}\left(\mathbb{R}^{N}\right)$ be the subspace of $L^{p}\left(\mathbb{R}^{n}\right)$ defined by

$$
\mathscr{D}^{p}\left(\mathbb{R}^{N}\right):=\left\{f \in L^{p}\left(\mathbb{R}^{N}\right) ; \operatorname{supp}(\hat{f}) \text { is compact }\right\},
$$

where $\hat{f}:=\mathscr{F}(f)$ is the Fourier transform of $f$ considered in the sense of distributions. The operator $S_{\phi}$ defined by $D\left(S_{\phi}\right):=\mathscr{D}^{p}\left(\mathbb{R}^{N}\right)$ and $S_{\phi} f:=\mathscr{F}^{-1}(\phi \hat{f})$, for every $f \in \mathscr{D}^{p}\left(\mathbb{R}^{N}\right)$, is closable; its closure is denoted by $S_{\phi}^{p}$. For $1<p \leqq 2$ it turns out that

$$
D\left(S_{\phi}^{p}\right)=\left\{f \in L^{p}\left(\mathbb{R}^{N}\right) ; \phi \hat{f} \in \mathscr{F}\left(L^{p}\left(\mathbb{R}^{N}\right)\right) \subset L^{q}\left(\mathbb{R}^{N}\right)\right\}
$$

and $S_{\phi}^{p} f=\mathscr{F}^{-1}(\phi \hat{f})$, for every $f \in D\left(S_{\phi}^{p}\right)$, where $p^{-1}+q^{-1}=1$. Using the fact that an element of $\mathscr{L}\left(L^{p}\left(\mathbb{R}^{N}\right)\right)$ is a $p$-multiplier operator if and only if it commutes with all translations it follows that if $\lambda$ is in the resolvent set $\rho\left(S_{\phi}^{p}\right)$ of $S_{\phi}^{p}$, then necessarily $\left(\lambda 1-S_{\phi}^{p}\right)^{-1}$ is a $p$-multiplier operator. This observation implies that the essential range ess $\operatorname{ran}(\phi)$ of $\phi$ is always contained in $\sigma\left(S_{\phi}^{p}\right)$. If, in addition, $S_{\phi}^{p}$ is also decomposable, then ess $\operatorname{ran}(\phi)=\sigma\left(S_{\phi}^{p}\right)$; see [2, Corollary 3.4].

For $k \in \mathbb{N}_{0}=\mathbb{N} \cup\{0\}$, let $\mathscr{A}^{k}$ denote the algebra of all functions $\phi \in \mathscr{C}^{k}(\mathbb{C})$ satisfying (with $x=x_{1}+i x_{2} \in \mathbb{C}$ identified with $\left(x_{1}, x_{2}\right) \in \mathbb{R}^{2}$ ),

$$
\|\phi\|_{\mathcal{A}^{\alpha}}:=\sum_{|\alpha| \leq k} \alpha !^{-1} \sup _{x \in \mathrm{C}}(1+|x|)^{|\alpha|} \cdot\left|\frac{\partial^{\alpha} \phi}{\partial x^{\alpha}}(x)\right|<\infty,
$$

where we use standard multi-index notation and $\alpha \in \mathbb{N}_{0}^{2}$. Endowed with this norm $\mathscr{A}^{k}$ is 
a commutative, semisimple Banach algebra with unit which has the following two properties.

(A) For every open cover $\left\{U_{1}, \ldots, U_{r}\right\}$ of $\mathbb{C}$ there are elements $\phi_{1}, \ldots, \phi_{r}$ in $\mathscr{A}^{k}$ (having continuous extensions at $\infty$ ) such that supp $\phi_{j} \subset U_{j}$, for $j=1, \ldots, r$, and $\phi_{1}+\cdots+\phi_{r} \equiv 1$ on $\mathbb{C}$.

(B) For every $\phi \in \mathscr{A}^{k}$ and every $\lambda \in \mathbb{C} \backslash \operatorname{supp} \phi$ the function

$$
z \mapsto \phi_{\lambda}(z)=\phi(z) /(\lambda-z)
$$

is in $\mathscr{A}^{k}$ and vanishes at $\infty$.

Let $N$ and $k$ be positive integers. Denote by $\mathcal{N}^{k}\left(\mathbb{R}^{N}\right)$ the family of all functions $\psi \in \mathscr{C}^{k}\left(\mathbb{R}^{N} \backslash\{0\}\right)$ which satisfy the condition

$$
\|\psi\|_{\mathcal{N}^{k}}\left(\mathbb{R}^{N}\right):=\sum_{|\beta| \leqq k} \beta !^{-1} \sup _{\xi \neq 0}|\xi||\beta| \cdot\left|\frac{\partial^{\beta} \psi}{\partial x^{\beta}}(\xi)\right|<\infty
$$

where $\beta \in \mathbb{N}_{0}^{N}$ and $|\xi|$ denotes the usual Euclidean norm on $\mathbb{R}^{N}$. Endowed with this norm $\mathcal{N}^{k}\left(\mathbb{R}^{N}\right)$ is a commutative, semisimple Banach algebra with unit. If $k>N / 2$ then the classical Mihlin multiplier theorem $\left[12\right.$, p. 96], ensures that $\mathcal{N}^{k}\left(\mathbb{R}^{N}\right)$ is continuously imbedded in $\mathscr{M}^{p}\left(\mathbb{R}^{N}\right)$, for every $1<p<\infty$.

The following fact can be established by induction.

Lemma 1.2. Let $k$ and $N$ be positive integers and $\Omega \subseteq \mathbb{R}^{N}$ be an open set. Let $\phi \in \mathscr{C}^{k}(\mathbb{C})$ and $\psi \in \mathscr{C}^{k}(\Omega)$. Identify $\mathbb{C}$ with $\mathbb{R}^{2}$ and write $x=\left(x_{1}, x_{2}\right)$ for points in $\mathbb{C}$. Denote $\psi$ by $\left(\psi_{1}, \psi_{2}\right)$ where $\psi_{1}=\operatorname{Re} \psi$ and $\psi_{2}=\operatorname{Im} \psi$. Let $\beta \in \mathbb{N}_{0}^{N}$ satisfy $|\beta| \leqq k$. Then, for each $\alpha \in \mathbb{N}_{0}^{2}$ satisfying $|\alpha| \leqq|\beta|$, there exist numbers $c_{\alpha} \in\{0,1\}$ and $r(\alpha, \beta) \in \mathbb{N}$, integers $\eta(j, \alpha, m) \in\{1,2\}$ and multi-indices $\gamma(j, \alpha, m) \leqq \beta$ (co-ordinatewise), for $1 \leqq j \leqq|\alpha|$ and $1 \leqq m \leqq r(\alpha, \beta)$, satisfying $\beta=\sum_{j=1}^{|\alpha|} \gamma(j, \alpha, m)$ such that

$$
\frac{\partial^{\beta}(\phi \circ \psi)}{\partial \xi^{\beta}}(\xi)=\sum_{|\alpha| \leqq|\beta|} c_{\alpha} \frac{\partial^{\alpha} \phi}{\partial x^{\alpha}}(\psi(\xi)) \cdot \sum_{m=1}^{r(\alpha, \beta)} \prod_{j=1}^{|\alpha|} \frac{\partial^{\gamma(j, \alpha, m)} \psi_{\eta(j, \alpha, m)}}{\partial \xi^{\gamma(j, \alpha, m)}}(\xi)
$$

for every $\xi \in \mathbf{\Omega}$.

We can now establish some facts about the algebras $\mathscr{A}^{k}$ and $\mathscr{N}^{k}\left(\mathbb{R}^{N}\right)$ which are needed later but which may also be of independent interest. For the definition of an operator $T \in \mathscr{L}(X)$ being generalized scalar we refer to the monograph [3].

Lemma 1.3. (i) Let $k \in \mathbb{N}_{0}$ and $\psi \in \mathscr{A}^{k}$.

(a) The multiplication operator $M_{\psi}: f \mapsto \psi f$, for $f \in \mathscr{A}^{k}$, is generalized scalar in $\mathscr{L}\left(\mathscr{A}^{k}\right)$.

(b) The Banach algebra $\mathscr{A}^{k}$ is regular. 
(ii) Let $k$ and $N$ be positive integers and $\phi \in \mathscr{N}^{k}\left(\mathbb{R}^{N}\right)$.

(a) The multiplication operator $M_{\psi}: g \mapsto \psi g$, for $g \in \mathcal{N}^{k}\left(\mathbb{R}^{N}\right)$, is generalized scalar in $\mathscr{L}\left(\mathscr{N}^{k}\left(\mathbb{R}^{N}\right)\right)$

(b) The Banach algebra $\mathscr{N}^{k}\left(\mathbb{R}^{N}\right)$ is regular.

(c) If $k>1 / 2$, then for each $1<p<\infty$, the (bounded) p-multiplier operator $S_{\phi}^{p}$ is generalized scalar in $\mathscr{L}\left(L^{p}\left(\mathbb{R}^{N}\right)\right)$.

Proof. (i) To establish (a) it suffices to show that $\rho \mapsto \rho \circ \psi$ defines a continuous homomorphism from $\mathscr{C}^{K}(\mathbb{C})$ into $\mathscr{A}^{k}$. This follows from Lemma 1.2 (by direct computation). Part (b) follows from (a) and [5, Theorem 2], after noting that generalized scalar operators are decomposable.

Parts (a) and (b) of (ii) can be proved similarly to those in (i). Finally, (c) follows from (a) and continuity of the canonical imbedding of $\mathscr{N}^{K}\left(\mathbb{R}^{N}\right)$ into $\mathscr{M}^{p}\left(\mathbb{R}^{N}\right)$.

Given a positive integer $k$, let $\mathscr{H}^{k}$ denote the space of all functions $h \in \mathscr{C}(\mathbb{C}) \cap$ $\mathscr{C}^{2 k}(\mathbb{C} \backslash\{0\})$ satisfying

$$
\|h\|_{\varkappa^{k}}:=\sum_{\beta \leqq(k, k)} \beta !^{-1} \sup _{\xi \in \mathbf{R}^{2}}\left|\xi^{\beta} \cdot \frac{\partial^{\beta} h}{\partial \xi^{\beta}}(\xi)\right|<\infty .
$$

Endowed with this norm $\mathscr{H}^{k}$ is a unital, commutative, semisimple Banach algebra. The following can be established along the lines of the proof of Lemma 1.3(i).

Lemma 1.4. Let $k \in \mathbb{N}$ and $\psi \in \mathscr{H}^{k}$.

(a) The multiplication operator $M_{\psi}: f \mapsto \psi f$, for $f \in \mathscr{H}^{k}$, is generalized scalar in $\mathscr{L}\left(\mathscr{H}^{k}\right)$.

(b) $\mathscr{H}^{k}$ is a regular Banach algebra.

Given $k \in \mathbb{N}$, let $Z_{k}:=\bigcup_{j=1}^{k}\left\{x \in \mathbb{R}^{k} ; x_{j}=0\right\}$. Let $\beta \in \mathbb{N}_{0}^{k}$ be a multi-index satisfying $|\beta|=k$. Then $\mathscr{L}^{\beta}\left(\mathbb{R}^{k}\right)$ denotes the space of all functions $\psi \in \mathscr{C}^{k}\left(\mathbb{R}^{k} \backslash Z_{k}\right)$ such that

$$
\|\psi\|_{\beta}:=\sum_{\alpha \leqq \beta} \alpha !^{-1} \sup _{\xi \notin Z_{k}}\left|\xi^{\alpha} \cdot \frac{\partial^{\alpha} \psi}{\partial \xi^{\alpha}}(\xi)\right|<\infty .
$$

Equipped with this submultiplicative norm $\mathscr{L}^{\beta}\left(\mathbb{R}^{k}\right)$ is a unital, commutative, normed algebra.

Lemma 1.5. (i) Let $k \in \mathbb{N}$ and $\beta \in \mathbb{N}_{0}^{k}$ satisfy $|\beta|=k$. Fix $\phi \in \mathscr{L}^{\beta}\left(\mathbb{R}^{k}\right)$. For each $\rho \in \mathscr{C}^{k}(\mathbb{C})$ the composition $\rho \circ \phi \in \mathscr{L}^{\beta}\left(\mathbb{R}^{k}\right)$ and the homomorphism $\rho \mapsto \rho \circ \phi$ is continuous from the Fréchet algebra $\mathscr{C}^{k}(\mathbb{C})$ into $\mathscr{L}^{\beta}\left(\mathbb{R}^{k}\right)$.

(ii) Let $\beta:=(1, \ldots, 1)$. Then each $\psi \in \mathscr{L}^{\beta}\left(\mathbb{R}^{k}\right)$ belongs to $\mathscr{H}^{p}\left(\mathbb{R}^{k}\right)$, for every $1<p<\infty$, and 
the canonical inclusion of $\mathscr{L}^{\beta}\left(\mathbb{R}^{k}\right)$ into $\mathscr{M}^{p}\left(\mathbb{R}^{k}\right)$ is continuous. Moreover, the (bounded) p-multiplier operator $S_{\phi}^{p}$ is generalized scalar in $\mathscr{L}\left(L^{p}\left(\mathbb{R}^{N}\right)\right)$.

Proof. (i) follows from the definition of the norm $\|\cdot\|_{\beta}$ and Lemma 1.2.

(ii) The first claim follows from Theorems 4.4 and 4.5 of [9]; put there $d=r=k$ and let $P: \mathbb{R}_{x}^{d} \rightarrow \mathbb{R}_{\xi}^{r}$ be the identity transformation. To establish the second claim it suffices to show that the map $\rho \mapsto S_{\rho \circ \psi}^{p}$ is continuous. This follows from (i) and the continuity of the homomorphism $g \mapsto S_{g}^{p}$ from $\mathscr{M}^{p}\left(\mathbb{B}^{k}\right)$ into $\mathscr{L}\left(L^{p}\left(\mathbb{R}^{k}\right)\right)$.

\section{Main results}

It was shown in [2] that elliptic differential operators with constant coefficients are always decomposable in $L^{p}\left(\mathbb{R}^{N}\right), 1<p<\infty$, and have a rich functional calculus. This is not the case in general [2, Corollary 3.5]. In this section we wish to exhibit further classes of multiplier operators which are decomposable. We begin with a result which is an extended version of Theorem 3.1 in [2] and shows that "elliptic" multiplier operators have a functional calculus rich enough to ensure that they are decomposable.

Theorem 2.1. Let $p \in(1, \infty)$. Let $k$ and $N$ be positive integers such that $k>N / 2$ and $\psi \in \mathscr{C}^{k}\left(\mathbb{R}^{N} \backslash\{0\}\right)$. Assume, for some $m \geqq 0$, that the following conditions are satisfied.

(i) $1 /|\psi(\xi)|=O\left(|\xi|^{-m}\right)$ for $|\xi| \rightarrow \infty$.

(ii) For all $\gamma \in \mathbb{N}_{0}^{N}$ with $|\gamma| \leqq k$ we have $\left|\frac{\partial^{\gamma} \psi}{\partial \xi^{\gamma}}(\xi)\right|=O\left(|\xi|^{m-|\gamma|}\right)$ for $|\xi| \rightarrow \infty$.

(iii) For all $\gamma \in \mathbb{N}_{0}^{N}$ with $|\gamma| \leqq k$ we have $\left|\frac{\partial^{\gamma} \psi}{\partial \xi^{\gamma}}(\xi)\right|=O\left(|\xi|^{-|\gamma|}\right)$ for $|\xi| \rightarrow 0$.

Then, for every $\phi \in \mathscr{A}^{k}$, the function $\phi \circ \psi$ is a p-multiplier on $\mathbb{R}^{N}$ and the homomorphism $\Phi: \mathscr{A}^{k} \rightarrow \mathscr{L}\left(L^{p}\left(\mathbb{R}^{N}\right)\right)$ defined by $\Phi(\phi):=S_{\phi \circ \psi}^{p}$, for $\phi \in \mathscr{A}^{k}$, is continuous and has the following properties.

(a) For all compactly supported elements $\phi \in \mathscr{A}^{k}$,

$$
\Phi(\phi) S_{\psi}^{p} \subseteq S_{\psi}^{p} \Phi(\phi)=\Phi\left(\phi \cdot i d_{\mathbf{c}}\right) .
$$

(b) There exists a sequence $\left(\rho_{n}\right)_{n=1}^{\infty}$ of functions in $\mathscr{A}^{k}$ with compact supports such that

$$
\Phi\left(\rho_{n}\right) \rightarrow 1 \text {, as } n \rightarrow \infty \text { with respect to the strong operator topology. }
$$


Moreover,

$$
D\left(S_{\psi}^{p}\right)=\left\{f \in L^{P}\left(\mathbb{R}^{N}\right) ; \lim _{n \rightarrow \infty} \Phi\left(\rho_{n} \cdot i d_{\mathrm{c}}\right) f \text { exists in } L^{p}\left(\mathbb{R}^{N}\right)\right\}
$$

and

$$
S_{\psi}^{p} f=\lim _{n \rightarrow \infty} \Phi\left(\rho_{n} \cdot i d_{c}\right) f, \quad f \in D\left(S_{\psi}^{p}\right)
$$

(c) The closed linear operator $S_{\psi}^{p}$ is decomposable and has the Ljubic-Macaev property. Moreover, $\sigma\left(S_{\psi}^{p}\right)=\overline{\psi\left(\mathbb{R}^{N} \backslash\{0\}\right)}=\operatorname{supp}(\Phi)$, where the bar denotes closure in $\overline{\mathbb{C}}$.

(d) Every operator $S_{\phi \circ \psi}^{p} \in \mathscr{L}\left(L^{p}\left(\mathbb{R}^{N}\right)\right)$, for $\phi \in \mathscr{A}^{k}$, is generalized scalar. In particular, for every $\lambda \in \rho\left(S_{\psi}^{p}\right)$, the operator $\left(\lambda 1-S_{\psi}^{p}\right)^{-1}$ is generalized scalar.

Proof. For $\alpha \in \mathbb{N}_{0}^{2}$ with $|\alpha| \leqq k$ it follows from the definition of $\mathscr{A}^{k}$ that

$$
|\psi(\xi)|^{|\alpha|}\left|\frac{\partial^{\alpha} \phi}{\partial x^{\alpha}}(\psi(\xi))\right| \leqq C\|\phi\|_{\alpha^{k}}, \quad \xi \in \mathbb{R}^{N} \backslash\{0\},
$$

for some constant $C>0$. Hence, for $\beta \in \mathbb{N}_{0}^{N}$ with $|\beta| \leqq k$, we obtain from assumptions (i) and (ii) and Lemma 1.2 that there exist constants $R>0$ and $C^{\prime}>0$ such that

$$
|\xi||\beta|\left|\frac{\partial^{\beta}(\phi \circ \psi)}{\partial \xi^{\beta}}(\xi)\right| \leqq C^{\prime}\|\phi\|_{\mathscr{A}^{k}}, \quad|\xi|>R .
$$

On the other hand, by assumption (iii) and Lemma 1.2 this inequality also holds in a deleted neighbourhood of 0 in $\mathbb{R}^{N}$ (with a possibly larger constant $C^{\prime \prime}$ than $C^{\prime}$ ). Since $\psi$ maps compact subsets of $\mathbb{R}^{N} \backslash\{0\}$ into compact subsets of $\mathbb{C}$ we conclude (again via Lemma 1.2) that (2) holds in $\mathbb{R}^{N} \backslash\{0\}$ (with a constant $C_{0}$ possibly larger than $C^{\prime}$ and $C^{\prime \prime}$ but still independent of $\phi$ ). By the Mihlin multiplier theorem [12, p. 96], $\phi \circ \psi$ is a $p$ multiplier on $\mathbb{R}^{N}$ and

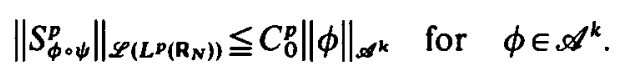

It follows that $\Phi: \mathscr{A}^{k} \rightarrow \mathscr{L}\left(L^{p}\left(\mathbb{R}^{N}\right)\right)$ is a continuous unital homomorphism. Then (a) follows, after noting that condition (i) implies that the range of $\Phi(\phi)$ is contained in $D\left(S_{\psi}^{p}\right)$ whenever $\operatorname{supp}(\phi)$ is compact.

To prove (b) fix any $\sigma \in \mathscr{C}_{c}^{\infty}(\mathbb{C})$ with $\sigma(0)=1$ and any $\mu>0$. Define

$$
\sigma_{n}: z \mapsto \sigma\left(n^{-\mu} z\right), \quad z \in \mathbb{C},
$$

for each $n \in \mathbb{N}$. Since $n^{-\mu} \leqq 1$ it follows from the definition of $\mathscr{A}^{k}$ that $\sigma_{n} \in \mathscr{A}^{k}$ and $\left\|\sigma_{n}\right\|_{\alpha^{k}} \leqq\|\sigma\|_{\alpha^{k}}$ for all $n \in \mathbb{N}$. Accordingly, $\left\{\Phi\left(\sigma_{n}\right) ; n \in \mathbb{N}\right\}$ is uniformly bounded in $\mathscr{L}\left(L^{p}\left(\mathbb{R}^{N}\right)\right)$. Since $\sigma_{n} \circ \psi \rightarrow 1$ pointwise in $\mathbb{R}^{N} \backslash\{0\}$, as $n \rightarrow \infty$, it follows that $\Phi\left(\sigma_{n}\right)=$ $S_{\sigma_{n} \circ \psi}^{p} \rightarrow 1$, as $n \rightarrow \infty$, with respect to the weak operator topology [6]. By Lemma 1.1 there is a sequence $\left(S_{n}\right)_{n=1}^{\infty}$ of the form

$$
S_{n}=\sum_{j=1}^{m(n)} \alpha_{j}^{(n)} \Phi\left(\sigma_{j}\right), \quad \text { with } \quad \alpha_{j}^{(n)} \geqq 0 \quad \text { satisfying } \quad \sum_{j=1}^{m(n)} \alpha_{j}^{(n)}=1,
$$


such that $S_{n} \rightarrow 1$, as $n \rightarrow \infty$, for the strong operator topology. This proves (1) with

$$
\rho_{n}:=\sum_{j=1}^{m(n)} \alpha_{j}^{(n)} \sigma_{j}
$$

The remaining statements in (b) can now be obtained from (1) and part (a) along the lines of the proof of Theorem 3.1 in [2].

(c) The subalgebra $\mathscr{B}^{k}$ of all $f \in \mathscr{A}^{k}$ having continuous extensions at $\infty$ is quasiadmissible in the sense of [14, Def. IV.9.2]. Hence, by the properties of $\Phi$ and [14, Corollary IV.9.8], the operator $S_{\psi}^{p}$ is decomposable. The Ljubic-Macaev property is obtained in the same way as for elliptic polynomials (c.f. the last part of the proof of Theorem 3.6 in [2]), as is the spectral mapping statement given in (c).

(d) As seen above, for each $\phi \in \mathscr{A}^{k}$, the function $\phi \circ \psi$ is in $\mathscr{N}^{k}\left(\mathbb{R}^{N}\right)$. Then Lemma 1.3 implies that $S_{\phi \circ \psi}^{p}$ is generalized scalar. Suppose now that $\lambda \in \rho\left(S_{\psi}^{p}\right)$. Let $\varepsilon>0$ be such that the closure of the open $\operatorname{disc} B_{\varepsilon}(\lambda)$ in $\mathbb{C}$ (centred at $\lambda$ and of radius $\varepsilon$ ) is contained in $\rho\left(S_{\psi}^{p}\right)$. Let $U \in \mathscr{C}^{\infty}(\mathbb{C})$ be any function such that $u \equiv 1$ in $\mathbb{C} \backslash B_{\varepsilon}(\lambda)$ and $u \equiv 0$ in $B_{\varepsilon / 2}(\lambda)$. Define $\phi(z):=u(z) /(\lambda-z)$ for $\lambda \in \mathbb{C}$. Then $\phi \in \mathscr{A}^{k}$ and $\phi(z) \equiv(\lambda-z)^{-1}$ in a neighbourhood of $\sigma\left(S_{\psi}^{p}\right)=\operatorname{supp}(\Phi)$. Accordingly, $\Phi(\phi)$ is generalized scalar. But, $\Phi(\phi)=S_{\phi \circ \psi}^{p}=$ $\left(\lambda 1-S_{\phi}^{p}\right)^{-1}$.

Remark. (i) The original version of the above result, formulated for elliptic polynomials of degree $m$ (c.f. [2, Theorems 3.1 and 3.6]) contained an algebra $\mathscr{A}_{m}^{k}$ depending on $m$. The present proof shows that we can always replace $\mathscr{A}_{m}^{k}$ by the larger algebra $\mathscr{A}^{k}$.

(ii) Examples of functions satisfying conditions (i)-(iii) of Theorem 2.1, besides elliptic polynomials, include all functions of the form $\psi=f_{1}+\cdots+f_{r}$, where $f_{j}$ is a homogeneous function in $\mathscr{C}^{k}\left(\mathbb{R}^{N} \backslash\{0\}\right)(k>N / 2)$ of order $v_{j} \geqq 0$, with $v_{j}<v_{r}$ for all $1 \leqq j<r$, and $f_{r}$ has no zeros in $\mathbb{R}^{N} \backslash\{0\}$. For instance, this includes the local $p$-multiplier $\psi(\xi)=|\xi|^{\alpha}$, for any $\alpha>0$, and hence in particular, includes the generator of the Poisson semigroup (put $\alpha=1$ ).

It is easy to exhibit local multipliers (even polynomials) which do not fall into the scheme covered by Theorem 2.1. The wave operator in 2-dimensions, whose symbol is $x^{2}-y^{2}$, is such an example. We wish now to suggest an approach which treats a class of operators of the form $Q(D)$ where $Q=Q_{1}+i Q_{2}$ is a polynomial in $\mathbb{R}^{N}$ (with $Q_{j}$ being $\mathbb{R}$-valued) such that both $Q_{1}$ and $Q_{2}$ can be factorized into the product of real affine functionals. The underlying technique is a modification of a multiplier result of Littman, McCarthy and Rivière, [9, Theorem 4.6].

Theorem 2.2. Let $p \in(1, \infty)$ and let $k$ and $N$ be positive integers. Let $l_{r}: \mathbb{R}^{N} \rightarrow \mathbb{R}$ and $m_{r}: \mathbb{R}^{N} \rightarrow \mathbb{R}$, for $1 \leqq r \leqq k$, be affine functionals and define

$$
Q(x):=\prod_{r=1}^{k} l_{r}(x)+i \cdot \prod_{r=1}^{k} m_{r}(x), \text { for } \quad x \in \mathbb{R}^{N}
$$


Then, for each $\phi \in \mathscr{H}^{k}$, the function $\phi \circ Q$ is a p-multiplier on $\mathbb{R}^{N}$ and the homomorphism $\Phi: \mathscr{H}^{k} \rightarrow \mathscr{L}\left(L^{p}\left(\mathbb{R}^{N}\right)\right)$ given by $\Phi(\phi):=S_{\phi . Q}^{p}$, for $\phi \in \mathscr{H}^{k}$, is continuous and has the following properties.

(a) For all $\phi \in \mathscr{H}^{k}$ with compact support,

$$
\Phi(\phi) S_{Q}^{p} \subseteq S_{Q}^{p} \Phi(\phi)=\Phi\left(\phi \cdot i d_{\mathrm{c}}\right) .
$$

(b) There exists a sequence $\left(\rho_{n}\right)_{n=1}^{\infty}$ of functions in $\mathscr{C}_{c}^{\infty}(\mathbb{C}) \subset \mathscr{H}^{k}$ such that $\Phi\left(\rho_{n}\right) \rightarrow 1$ as $n \rightarrow \infty$, in the strong operator topology. With this sequence we have

$$
D\left(S_{Q}^{p}\right)=D_{p}:=\left\{f \in L^{p}\left(\mathbb{R}^{N}\right) ; \lim _{n \rightarrow \infty} \Phi\left(\rho_{n} \cdot i d_{c}\right) f \text { exists in } L^{p}\left(\mathbb{R}^{N}\right)\right\}
$$

and

$$
S_{Q}^{p} f=\lim _{n \rightarrow \infty} \Phi\left(\rho_{n} \cdot i d_{\mathrm{c}}\right) f, \quad f \in D\left(S_{Q}^{p}\right)
$$

(c) The differential operator $Q(D)=S_{Q}^{p}$ is decomposable in $L^{p}\left(\mathbb{R}^{N}\right)$, has the LjubicMacaev property and satisfies $\sigma\left(S_{Q}^{p}\right)=\overline{Q\left(\mathbb{R}^{N}\right)}=\operatorname{supp}(\Phi)$.

(d) Every operator $S_{\phi \cdot Q}^{p} \in \mathscr{L}\left(L^{p}\left(\mathbb{R}^{N}\right)\right)$, for $\phi \in \mathscr{H}^{k}$, is generalized scalar. In particular, the operator $\left(\lambda 1-S_{Q}^{p}\right)^{-1}$ is generalized scalar whenever $\lambda \in \rho\left(S_{Q}^{p}\right)$.

Proof. We proceed as in the proof of Theorem 4.6 in [9]. Consider the affine transformation from $\mathbb{R}^{N}$ into $\mathbb{R}^{2 k}$ given by $\xi_{r}=l_{r}(x)$ and $\xi_{k+r}=m_{r}(x)$, for $1 \leqq r \leqq \mathrm{k}$. Define $F(\xi)=\phi\left(\psi_{1}(\xi), \psi_{2}(\xi)\right)$, for $\xi \in \mathbb{R}^{2 k}$, where $\psi_{1}(\xi):=\prod_{r=1}^{k} \xi_{r}$ and $\psi_{2}(\xi):=\prod_{r=1}^{k} \xi_{k+r}$, and $\phi$ is considered as a function in $\mathbb{R}^{2} \cong \mathbb{C}$. That is, $F=\phi \circ \psi$ where $\psi=\left(\psi_{1}, \psi_{2}\right)$ maps $\mathbb{R}^{2 k}$ into $\mathbb{R}^{2} \cong \mathbb{C}$. As in the proof of Theorem 4.6 in [9], it suffices to show that

$$
\left|\xi^{\alpha} \frac{\partial^{\alpha} F}{\partial \xi^{\alpha}}(\xi)\right| \leqq B_{p}\|\phi\|_{\boldsymbol{x}^{k},}, \quad \xi \in \mathbb{R}^{2 k}
$$

for any $2 k$-tuple $\alpha$ consisting of zeros and ones only. It follows from the chain rule and an inductive argument that, for every such $\alpha$,

$$
\xi^{\alpha} \frac{\partial^{\alpha} F}{\partial \xi^{\alpha}}(\xi)=\sum_{(j, r) \leqq(u, v)} C_{j, r} \psi_{1}(\xi)^{j} \psi_{2}(\xi)^{r} \frac{\partial^{j+r} \phi}{\partial s^{j} \partial t^{r}}(\psi(\xi)), \quad \xi \in \mathbb{R}^{2 k},
$$

where $u=\sum_{m=1}^{k} \alpha_{m}$ and $v=\sum_{m=1}^{k} \alpha_{k+m}$, and the $C_{j, r}$ are constants depending only on $\alpha$ and $k$. From the assumption that $\phi \in \mathscr{H}^{k}$ it follows that (3) is satisfied and hence $\phi \circ Q \in \mathscr{M}^{p}\left(\mathbb{R}^{N}\right)$. Moreover, (3) also establishes the estimate

$$
\|\Phi(\phi)\|=\left\|S_{\phi \cdot Q}^{p}\right\| \leqq B\|\phi\|_{\mathscr{X}^{k}}, \quad \phi \in \mathscr{H}^{k},
$$

where $B$ only depends on $N, k$ and $p$; see Theorems 4.4 and 4.5 in [9]. 
(a) A "Banach-Saks type argument" along the lines of the proof of Theorem 2.1(b) shows that there exists a sequence $\left(\chi_{m}\right)_{m=1}^{\infty}$ in $\mathscr{C}_{c}^{\infty}\left(\mathbb{R}^{N}\right)$ such that $S_{\chi m}^{p} \rightarrow 1$, as $m \rightarrow \infty$, in the strong operator topology. Note that the range of $S_{x m}^{p}$ is contained in $D\left(S_{Q}^{p}\right)$, for all $m \in \mathbb{N}$. For $f \in D\left(S_{Q}^{p}\right)$ we have

$$
S_{x m}^{p} \Phi(\phi) S_{Q}^{p} f=\Phi(\phi) S_{Q}^{p} S_{x m}^{p} f=S_{x m}^{p} \Phi\left(\phi \cdot i d_{\mathbb{C}}\right) f=S_{Q}^{p} S_{x m}^{p} \Phi(\phi) f
$$

Since the left-hand-side of this identity converges to $\Phi(\phi) S_{Q}^{p} f$, as $m \rightarrow \infty$, and $S_{\chi m}^{p} \Phi(\phi) f \rightarrow \Phi(\phi) f$, as $m \rightarrow \infty$, the closedness of $S_{Q}^{p}$ implies that $\Phi(\phi) f \in D\left(S_{Q}^{p}\right)$ and

$$
\Phi(\phi) S_{Q}^{p} f=S_{Q}^{p} \Phi(\phi) f=\Phi\left(\phi \cdot i d_{c}\right) f
$$

(b) The functions $\left(\rho_{n}\right)_{n=1}^{\infty}$ are constructed in the same way as in the proof of Theorem 2.1(b) to deduce that $\Phi\left(\rho_{n}\right) \rightarrow 1$, as $n \rightarrow \infty$, in the strong operator topology. The inclusion $D\left(S_{Q}^{p}\right) \subseteq D_{p}$ is obtained as in the proof of Theorem 3.1 in [2]. The converse inclusion is not so obvious as in Theorem 2.1. Fix $f \in D_{p}$ and let $\left(\chi_{m}\right)_{m=1}^{\infty}$ be as in the proof of (a). Then, for each $m \in \mathbb{N}$, we have $S_{\chi_{m}}^{p} f \in D_{p}$ and

$$
\lim _{n \rightarrow \infty} \Phi\left(\rho_{n} \cdot i d_{\mathbb{C}}\right) S_{\chi_{m}}^{p} f=S_{\chi_{m}}^{p} \lim _{n \rightarrow \infty} \Phi\left(\rho_{n} \cdot i d_{\mathbb{C}}\right) f
$$

The left-hand-side of this equation is also equal to

$$
\lim _{n \rightarrow \infty} S_{\rho_{n} \circ Q}^{p} S_{Q}^{p} S_{\chi_{m}}^{p} f=S_{Q}^{p} S_{\chi_{m}}^{p} f
$$

Letting now $m \rightarrow \infty$ and using the closedness of $S_{Q}^{p}$ it follows that $f \in D\left(S_{Q}^{p}\right)$ and

$$
S_{Q}^{p} f=\lim _{n \rightarrow \infty} \Phi\left(\rho_{n} \cdot i d_{c}\right) f
$$

To establish (c) observe that the algebra $\mathscr{K}^{k}$ of all functions in $\mathscr{H}^{k}$ having continuous extensions at $\infty$ is again quasiadmissible and then proceed as in the proof of Theorem 2.1.(c).

(d) By (3) we have $\phi \circ Q \in \mathscr{L}^{\beta}\left(\mathbb{R}^{N}\right)$ with $\beta=(1, \ldots, 1)$. Hence, Lemma 1.5 (ii) shows that $\Phi(\phi)$ is generalized scalar whenever $\phi \in \mathscr{H}^{k}$. The remainder of the argument follows along the lines of the proof of Theorem 2.1.(d).

It follows from Theorem 2.2 that the wave operator

$$
\frac{\partial^{2}}{\partial x^{2}}-\frac{\partial^{2}}{\partial y^{2}}
$$

in two dimensions is decomposable in $L^{p}\left(\mathbb{R}^{2}\right)$, for all $1<p<\infty$; put $l_{1}(x, y)=x+y$, $l_{2}(x, y)=y-x$ and $m_{1}=m_{2} \equiv 0$. Similarly 


$$
\frac{\partial^{2}}{\partial x^{2}}-\frac{\partial^{2}}{\partial y^{2}}+\frac{\partial}{\partial x}
$$

is decomposable in $L^{p}\left(\mathbb{R}^{2}\right)$; put $l_{1}$ and $l_{2}$ as above and $m_{1}(x, y)=x$ and $m_{2} \equiv-1$. So, Theorem 2.2 applies to a different class of differential operators than Theorem 2.1.

We now suggest a third result which applies to yet another class of multiplier operators. First we need some further notation.

Let $N$ be a positive integer and write $e:=(1, \ldots, 1) \in \mathbb{N}^{N}$. For any multi-index $\beta \leqq e$ we define $\mathbb{R}^{\beta}:=\left\{x \in \mathbb{R}^{N} ; x_{j}=0\right.$ if $\left.\beta_{j}=0,1 \leqq j \leqq N\right\}$ and consider $\mathbb{R}^{\beta}$ as a subspace of $\mathbb{R}^{N}$. For $x \in \mathbb{R}^{N}$ we denote by $x_{\beta} \in \mathbb{R}^{\beta}$ the canonical projection of $x$ into $\mathbb{R}^{\beta}$, that is

$$
\left(x_{\beta}\right)_{j}=\left\{\begin{array}{lll}
0 & \text { if } & \beta_{j}=0 \\
x_{j} & \text { if } & \beta_{j}=1
\end{array}\right.
$$

A bounded function $m=\mathscr{C}^{N}\left(\mathbb{R}^{N} \backslash Z_{N}\right)$ is said to have the Marcinkiewicz property if there exists a constant $B>0$ such that $\|m\|_{\infty} \leqq B$ and for each non-zero $\beta \leqq e$,

$$
\sup _{x_{\varepsilon-\beta} \rho}\left|\frac{\partial^{\beta} m}{\partial x^{\beta}}\right| d x_{\beta} \leqq B
$$

for every dyadic rectangle $\rho$ of $\mathbb{R}^{\beta}$ (see $[12$, p. 103], for the definition of dyadic rectangles). If $\beta=e$, the "sup" sign is omitted. The classical Marcinkiewicz multiplier theorem [12, p. 109], states that such a function $m$ belongs to $\mathscr{M}^{p}\left(\mathbb{R}^{N}\right)$, for every $1<p<\infty$. Moreover, an examination of the proof of Theorem $6^{\prime}$ in $[12$, p. 109], shows that $\left\|S_{m}^{p}\right\|_{\mathscr{L}\left(L^{\left.p\left(R^{N}\right)\right)} \leqq B\right.} C_{p, N}$, where $C_{p, N}$ is a constant only depending on $N$ and $p$. Let IImIl denote the infimum of all constants $B>0$ with the above property. Then also $B=\|m\|$ satisfies this property and so

$$
\left\|S_{m}^{p}\right\|_{\mathscr{L}\left(L^{\left.p\left(\mathbf{R}^{N}\right)\right)}\right.} \leqq C_{p, N} \cdot\|m\| .
$$

Moreover, $\|\cdot\|$ is a norm on the linear subspace of $\mathscr{C}^{N}\left(\mathbb{R}^{N} \backslash Z_{N}\right)$ consisting of all functions $m$ having the Marcinkiewicz property. Unlike the spaces of multipliers in the previous parts of this section, the Marcinkiewicz multipliers do not form an algebra.

Proposition 2.3. Let $\psi \in \mathscr{C}^{N}\left(\mathbb{R}^{N} \backslash Z_{N}\right)$ be a bounded function. Assume that there exists some constant $A>0$ such that, for each non-zero $\beta \leqq e$ and each partition $\beta=\gamma(1)+\cdots+\gamma(v), 1 \leqq v \leqq|\beta|$, with $\gamma(j)$ non-zero for all $j=1, \ldots, v$, we have

$$
\sup _{x_{e}-\beta \rho} \int_{j=1}^{\nu}\left|\prod_{j=1}^{\gamma(j)} \psi_{\eta(j)}\right| d x_{\beta}<A,
$$

for every dyadic rectangle $\rho$ of $\mathbb{P}^{\beta}$ and every choice of $\eta(1), \ldots, \eta(v) \in\{1,2\}$, where 
$\psi_{1}=\operatorname{Re} \psi$ and $\psi_{2}=\operatorname{Im} \psi$. Then, for any function $\phi \in \mathscr{C}^{N}(\mathscr{C})$, the function $\phi \circ \psi$ has the Marcinkiewicz property and satisfies

$$
\|\phi \circ \psi\| \leqq C_{0} q_{k}(\phi)
$$

where $C_{0}$ is a constant depending only on $A$ and $N$, and with $K:=\overline{\psi\left(\mathbb{R}^{N} \backslash Z_{N}\right)}$,

$$
q_{K}(\phi)=\max \left\{\sup _{z \in K}\left|\frac{\partial^{\alpha} \phi}{\partial z^{\alpha}}(z)\right| ; \alpha \in \mathbb{N}_{0}^{2},|\alpha| \leqq N\right\}
$$

In particular, $\phi \circ \psi \in \mathscr{M}^{p}\left(\mathbb{R}^{N}\right)$, for every $p \in(1, \infty)$, and

$$
\left\|S_{\phi \circ \psi}^{p}\right\|_{\mathscr{L}\left(L^{p}\left(\mathbf{R}^{N}\right)\right)} \leqq C_{p, N} C_{0} q_{K}(\phi) .
$$

The map $\Phi: \mathscr{C}^{N}(\mathbb{C}) \rightarrow \mathscr{L}\left(L^{p}\left(\mathbb{R}^{N}\right)\right)$ given by $\Phi(\phi):=S_{\phi \circ \psi}^{p}$, for $\phi \in \mathscr{C}^{N}(\mathbb{C})$, defines a $\mathscr{C}^{N}(\mathbb{C})$-functional calculus for $S_{\psi}^{p}$ and hence each operator $S_{\phi \circ \psi}^{p}, \phi \in \mathscr{C}^{N}(\mathbb{C})$, is generalized scalar, too.

Proof. The fact that $\phi \circ \psi$ has the Marcinkiewicz property and the estimate (5) follow by means of Lemma 1.2. By (4) and (5) we obtain (6) which implies the remaining statements.

We illustrate the use of Proposition 2.3 in some examples.

First consider $\psi(x, y)=|x|^{1 / 2}|y|^{1 / 2}\left(x^{2}+y^{2}\right)^{-1 / 2}$ which is an element of $\mathscr{C}^{2}\left(\mathbb{R}^{2} \backslash Z_{2}\right)$ satisfying the Marcinkiewicz property; see [12, p. 110]. Noting that $\operatorname{Im} \psi=0$ and $\psi$ is symmetric with respect to the variables $x$ and $y$, we see that the assumptions of Proposition 2.3 reduce to checking that

$$
\sup _{\rho}\left|\frac{\partial \psi}{\partial x}\right| \cdot\left|\frac{\partial \psi}{\partial y}\right| d x d y<\infty
$$

where the sup is taken over all dyadic rectangles in the positive quadrant of $\mathbb{R}^{2}$. Direct calculation shows that

$$
\left|\frac{\partial \psi}{\partial x}\right| \cdot\left|\frac{\partial \psi}{\partial y}\right| \leqq \frac{4}{x^{2}+y^{2}}
$$

Accordingly, for

$$
\rho=\left[2^{n}, 2^{n+1}\right] \times\left[2^{m}, 2^{m+1}\right] \text { or } \rho=\left[2^{-n-1}, 2^{-n}\right] \times\left[2^{-m-1}, 2^{-m}\right]
$$

the estimate (7) clearly holds. For "mixed" cases

$$
\rho=\left[2^{n}, 2^{n+1}\right] \times\left[2^{-m-1}, 2^{-m}\right]
$$


or the other possibility, a change to polar coordinates shows that (7) holds. So, the conditions of Proposition 2.3 are satisfied. Hence, the operator $S_{\psi}^{p}$ is generalized scalar, for every $1<p<\infty$.

For the next consequence of Proposition 2.3 we need the following general fact, which is probably known. For the sake of completeness we include a proof.

Lemma 2.4. Let $X$ be a Banach space and $T \in \mathscr{C}(X)$ be a closed, injective operator in $X$.

(a) For all closed sets $F \subseteq \overline{\mathbb{C}}$, we have $\mathscr{X}_{T}(F)=\mathscr{X}_{T^{-1}}\left(F^{-1}\right)$, where ${ }^{*} F^{-1}=\left\{z^{-1} ; z \in F\right\}$ with the convention $\infty^{-1}=0$ and $0^{-1}=\infty$.

(b) $T$ is decomposable if and only if $T^{-1}$ is decomposable.

Proof. (a) Fix an arbitrary element $x \in \mathscr{X}_{T}(F)$. Hence we have $x \equiv(z-T) f(z)$ on $\mathbb{C} \backslash F$, where $f$ is an analytic $X$-valued function on $\mathbb{\mathbb { C }} \backslash F$ with $f(z) \in D(T)$ for all $z \in \mathbb{C} \backslash F$. It follows that $x \equiv\left(z^{-1}-T^{-1}\right)(-z T f(z))$ on $\mathbb{C} \backslash F$. The $X$-valued function

$$
g: w \mapsto g(w):=-w^{-1} T f\left(w^{-1}\right) \equiv w^{-1}\left(x-w^{-1} f\left(w^{-1}\right)\right)
$$

is analytic on $\mathbb{C} \backslash\left(F^{-1} \cup\{0\}\right)$ with $g(w) \in D\left(T^{-1}\right)=\operatorname{ran}(T)$, for all $w \in \mathbb{C} \backslash\left(F^{-1} \cup\{0\}\right)$, and $\infty$ is a removable singularity if $0 \in F$. If $\infty \notin F$, we still have to show that 0 is a removable singularity for $g$. Notice that in this case, the limit

$$
\lim _{z \rightarrow \infty} T\left(z^{-1} f(z)\right)=\lim _{z \rightarrow \infty}\left(f(z)-z^{-1} x\right)=f(\infty) \in X
$$

exists on $X$. Since $T$ is a closed operator and $z^{-1} f(z) \rightarrow 0$ for $z \rightarrow \infty$ this shows that $f(\infty)=0$ (this fact is also contained in the proof of Satz 3.19 in [1]). The function $f$ being analytic at $\infty$, this implies that $\lim _{z \rightarrow \infty} z f(z)$ exists in $X$. Using again the fact that $T$ is closed we see that

$$
\lim _{z \rightarrow \infty} z f(z)=\lim _{z \rightarrow \infty}(T f(z)+x)=x .
$$

Therefore the function $h$ with

$$
h(w)=\left\{\begin{array}{lll}
x-w^{-1} f\left(w^{-1}\right) & \text { for } & w \in \mathbb{C} \backslash\left(F^{-1} \cup\{0\}\right) \\
0 & \text { for } & w=0
\end{array}\right.
$$

is analytic on $\mathbb{C} \backslash F^{-1}$. This shows that

$$
\lim _{w \rightarrow 0} g(w)=h^{\prime}(0)
$$

exists in $X$. In particular, 0 is a removable singularity of $g$. Moreover, from the 
closedness of the operator $T^{-1}$ and the equation $x \equiv\left(w-T^{-1}\right) g(w)$ on $\mathbb{C} \backslash\left(F^{-1} \cup\{0\}\right)$ we see that $g(0):=h^{\prime}(0) \in D\left(T^{-1}\right)$. Thus we have proved that $\mathscr{X}_{T}(F) \subseteq \mathscr{X}_{T^{-1}}\left(F^{-1}\right)$. The proof of the reverse inclusion is now obtained by interchanging $T$ with $T^{-1}$ and $F$ with $F^{-1}$ in the first part of the proof.

(b) Let $T$ be decomposable. A straight forward consideration using (a) and some basic facts from local spectral theory ([14, Sections IV.3 and IV.4] show that the map $F \rightarrow \mathscr{X}_{T^{-1}}\left(F^{-1}\right)$ then defines a spectral capacity for $T$. Hence, $T^{-1}$ is decomposable by [14, IV.4.26]. The converse implication is obtained by interchanging $T$ with $T^{-1}$ in these arguments.

Corollary 2.5. Let $p \in(1, \infty)$ and let $Q \in \mathscr{C}^{N}\left(\mathbb{R}^{N} \backslash Z_{N}\right) \cap \mathscr{U}^{p}\left(\mathbb{R}^{N}\right)$ be a function such that for some $\lambda \in \mathbb{C} \backslash Q\left(\mathbb{R}^{N} \backslash Z_{N}\right)$ the function $\psi(x)=(\lambda-Q(x))^{-1}$ satisfies the assumptions in Proposition 2.3. Then the (possibly unbounded) multiplier operator $S_{Q}^{p}$ is decomposable and hence satisfies $\sigma\left(S_{Q}^{p}\right)=\overline{Q\left(\mathbb{R}^{N} \backslash Z_{N}\right)}$.

Proof. By Proposition 2.3 the operator $S_{\psi}^{p}$ is bounded and generalized scalar. Since then $S_{\psi}^{p}=\left(\lambda-S_{Q}^{p}\right)^{-1}$ it follows from the preceding lemma that $S_{Q}^{p}$ is decomposable.

Corollary 2.5 is applicable to certain differential operators $Q(D)$ which do not satisfy the criteria of Theorems 2.1 and 2.2. The idea is to find a point $\lambda \in \mathbb{C}$ such that $(\lambda-Q(x))^{-1}$ satisfies the criteria of Proposition 2.3. For example, consider the differential operator

$$
-\frac{\partial^{2}}{\partial x^{2}}+\frac{\partial^{2}}{\partial y^{2}}+2 \frac{\partial}{\partial x}-2
$$

It is clearly not elliptic nor does its symbol $Q(x, y)=Q_{1}(x, y)+i Q_{2}(x, y)$, where $Q_{1}(x, y)=$ $x^{2}-y^{2}-2$ and $Q_{2}(x, y)=2 x$, have the property that both $Q_{1}$ and $Q_{2}$ factorize into a product of real affine functionals $\left(Q_{1}\right.$ is the problem). Consider the point $\lambda=0$ and let $\psi(x, y)=(\lambda-Q(x, y))^{-1}$. Then

$$
(\operatorname{Re} \psi)(x, y)=\frac{x^{2}-y^{2}-2}{\left(x^{2}-y^{2}-2\right)^{2}+4 x^{2}} \quad \text { and }(\operatorname{Im} \psi)(x, y)=\frac{-2 x}{\left(x^{2}-y^{2}-2\right)^{2}+4 x^{2}} \quad \text { for }(x, y) \in \mathbb{R}^{2}
$$

and hence, $\psi \in \mathscr{C}^{\infty}(\mathbb{C}) \cong \mathscr{C}^{\infty}\left(\mathbb{R}^{2}\right)$ with $\lim _{(x, y) \rightarrow \infty} \psi(x, y)=0$. Making the change of variables $s=x-y$ and $t=x+y$ we see that $\operatorname{Re} \psi$ and $\operatorname{Im} \psi$ belong to $\mathscr{M}^{p}\left(\mathbb{R}^{2}\right)$ if and only if the transformed functions

$$
\psi_{1}(s, t)=\frac{s t-2}{(s t-2)^{2}+(s+t)^{2}} \quad \text { and } \psi_{2}(s, t)=\frac{-s-t}{(s t-2)^{2}+(s+t)^{2}} \quad \text { for }(s, t) \in \mathbb{R}^{2}
$$

belong to $\mathscr{M}^{p}\left(\mathbb{R}^{2}\right)$. The Mihlin multiplier theorem does not apply as 


$$
\left(s^{2}+t^{2}\right)\left|\frac{\partial^{2} \psi_{1}(s, t)}{\partial s^{2}}\right|
$$

is not bounded. However, direct computation shows that both $\psi_{1}$ and $\psi_{2}$ satisfy the conditions of Proposition 2.3. So, Corollary 2.5 implies that $S_{\psi_{1}+i \psi_{2}}^{p}$ and hence, also $S_{\psi}^{p}=(\lambda 1-Q(D))^{-1}$ is generalized scalar. In particular, the (unbounded) differential operator $Q(D)$ is decomposable and the spectral mapping property $\sigma(Q(D))=\overline{Q\left(\mathbb{R}^{2}\right)}$ holds for every $1<p<\infty$.

We have restricted our attention to the particular case of local p-multipliers (often just polynomials) because in this setting it is possible to use a systematic approach to develop results (via classical multiplier theorems) which apply to large classes of $p$ multiplier operators. However, it should be noted that there exist relatively simple functions which may fail to be local multipliers (even bounded reciprocals of polynomials); see [10], for example. Such "multipliers", although not covered by the framework of this note, may still be "nice". For example, consider $\psi(x)=\ln |x|$, for $0 \neq x \in \mathbb{R}$. Then $\psi$ is not a local $p$-multiplier in our sense, for any $1<p<\infty$. Let $\Lambda(x)=(i+\ln |x|)^{-1}$, for $0 \neq x \in \mathbb{R}$. Then direct calculation shows that $\Lambda \in \mathcal{N}^{-1}(\mathbb{R})$. Accordingly, the resolvent operator $S_{(\psi+i)^{-1}}^{p}=S_{\Lambda}^{p}$ is generalized scalar and, in particular, $S_{\psi}^{p}$ is decomposable. A point which has been passed over, but actually needs checking, is that $S_{\psi}^{p}$ is closed and densely defined. This can be seen as follows (for $1<p \leqq 2$ ). Define $\phi_{t}(z)=e^{i t z}$, for $z \in \mathbb{C}$, in which case $\phi_{t} \in \mathscr{C}^{\infty}(\mathbb{C}) \cong \mathscr{C}^{\infty}\left(\mathbb{R}^{2}\right)$ for every $t \in \mathbb{R}$. Then $\phi_{t \circ \psi} \in \mathscr{C}^{\infty}(\mathbb{R} \backslash\{0\})$ and the estimates for the Mihlin theorem show that $\left\|\phi_{t \circ \psi}\right\|_{\mathcal{M}^{p}} \leqq C_{p}|t|, t \in \mathbb{R}$. So, $W_{t}=S_{\phi_{t}{ }^{\circ} \psi}^{p}$, for $t \in \mathbb{R}$, is a group of bounded operators in $L^{p}(\mathbb{R})$. Since $\phi t_{n} \circ \psi \rightarrow 1$ pointwise on $\mathbb{R} \backslash\{0\}$ as $n \rightarrow \infty$, whenever $t_{n} \rightarrow 0$, and $\sup \left\{\left\|W_{t_{n}}\right\| ; n \in \mathbb{N}\right\}<\infty$, it follows that $W_{t_{n}} \rightarrow 1$ in the weak operator topology as $n \rightarrow \infty$, [6]. By general semigroup theory it follows that actually $W_{t_{n}} \rightarrow 1$ in the strong operator topology as $n \rightarrow \infty,\left[7\right.$, Theorem 10.6.5]. That is $\left\{W_{t}\right\}_{t \in \mathbf{R}}$ is a strongly continuous $C_{0}$-group and hence, its infinitesimal generator (defined via general semigroup theory) is closed and densely defined. That this infinitesimal generator coincides with the multiplier operator $S_{i \psi}^{p}$ with its natural domain

$$
D\left(S_{i \psi}^{p}\right)=\left\{g \in L^{p}(\mathbb{R}) ; i \psi \hat{g}=\hat{h} \text { for some } h \in L^{p}(\mathbb{R})\right\},
$$

can be argued as in the proof of Theorem 21.4.2 in [7].

\section{REFERENCES}

1. E. Albrecht, Spektalmasße und Funktionalkalküle auf topologischen Vektorräumen (Diplomarbeit, Johannes Gutenberg-Universität Mainz, 1970).

2. E. Albrecht and W. J. Ricker, Local spectral properties of constant coefficient differential operators. In $L^{p}\left(\mathbf{R}^{N}\right), J$. Operator Theory 24 (1990), 85-103.

3. I. Colojoara and C. Folas, Theory of generalized spectral operators (Gordon and Breach, New York, 1968).

4. N. Dunford and J. T. Schwartz, Linear operators III: Spectral Operators (Wiley-Interscience, New York, 1971). 
5. ST. Frunza, A characterization of regular Banach algebras, Rev. Roumaine Math. Pures Appl. 18 (1973), 1057-1059.

6. G. I. Gaudry and W. J. Ricker, Spectral properties of $L^{p}$-translations, J. Operator Theory 14 (1985), 87-111.

7. E. Hille and R. S. Phillips, Functional analysis and semi-groups (American Math. Soc. Colloquium Publications Vol. XXXI, Amer. Math. Soc. Providence, R.I., 1957).

8. S. Kakutanl, Weak convergence in uniformly convex spaces, Tôhoku Math. J. 45 (1938), 188-193.

9. W. Littmann, C. McCarthy and N. Riviere, $L^{p}$-multiplier theorems, Studia Math. 30 (1968), 193-217.

10. W. Littmann, C. McCarthy and N. Riviere, The non-existence of $L^{p}$ estimates for certain translation invariant operators, Studia Math. 30 (1968), 219-229.

11. J. L. LuUbic and V. I. MaCAEv, On operators with separable spectrum, Mat. Sb. 56 (1962), 433-468.

12. E. M. Stein, Singular integrals and differentiability properties of functions (Princeton University Press, Princeton, N.J., 1970).

13. W. Szlenk, Sur les suites faiblement convergentes dans l'espace $L$, Studia Math. 25 (1965), 337-341.

14. F.-H. Vasilescu, Analytic functional calculus and spectral decompositions (D. Reidel Publ. Comp., Dordrecht, and Editura Academiei, Bucureşti, 1982).

Fachbereich Mathematik

UNIVERSität des SAARLANDES

Postrach 1150

D-66141 SaARbrucken

Germany
SCHOOl of Mathematics

University of New South Wales

P.O. Box 1

Kensington, N.W.S., 2033

Australia 